\title{
HOOK REPRESENTATIONS OF THE SYMMETRIC GROUPS
}

\author{
by M. H. PEEL \\ (Received 20 October, 1969)
}

1. Introduction. In this paper we are concerned with the representation theory of the symmetric groups over a field $K$ of characteristic $p$. Every field is a splitting field for the symmetric groups. Consequently, in order to study the modular representation theory of these groups, it is sufficient to work over the prime fields. However, we take $K$ to be an arbitrary field of characteristic $p$, since the presentation of the results is not affected by this choice. $S_{n}$ denotes the group of permutations of $\left\{x_{1}, \ldots, x_{n}\right\}$, where $x_{1}, \ldots, x_{n}$ are independent indeterminates over $K$. The group algebra of $S_{n}$ with coefficients in $K$ is denoted by $\Phi_{n}$.

Let $(\lambda)$ be a partition of $n$. We can define the Specht module $S^{(\lambda)}$ corresponding to $(\lambda)$ (over the field $K$ ). For the construction, the reader is referred to [3]. $S^{(\lambda)}$ consists of $K$-linear combinations of the polynomials $f^{(\lambda)}(y)$ defined for any tableau $y$ of $(\lambda)$ as in [3, Introduction]. These modules are studied in the author's Ph.D. thesis [4]. If $K$ is a field of characteristic zero, the Specht modules constitute a full set of irreducible, non-isomorphic left $\Phi_{n}$-modules.

It turns out that the problem of determining the $p$-decomposition numbers of the symmetric groups is equivalent to the problem of constructing the composition factors of the Specht modules over a field of characteristic $p$. We attempt to analyse the Specht modules corresponding to partitions of the form $\left(n-r, 1^{r}\right), 0 \leqq r \leqq n-1$. The diagram of such a partition is a hook, and accordingly, these Specht modules are referred to as Hook Representations. The Specht module corresponding to the partition $\left(n-r, 1^{r}\right)$ is denoted by $S(r, n)$.

Throughout most of this paper, $K$ is a field of characteristic $p$ not equal to 2 . We prove that $S(r, n)$ is irreducible if $p$ does not divide $n$. In the case in which $p$ divides $n$, we find a composition series of the form $0 \subset M \subset S(r, n), 0<r<n-1$. The proof is by induction on $n$. In the first few sections we lay the foundations of the inductive argument which is carried out in Section 5.

The paper is concluded in Section 6 with some remarks concerning the hook representations over a field of characteristic 2 . We establish a connection between these modules and the natural representation modules to be defined. If $2 r \leqq n, S(r, n)$ contains a submodule isomorphic to the Specht module corresponding to the partition $(n-r, r)$. The problem of analysing the hook representations is equivalent to that of analysing the natural representation modules.

For a general reference on the representations of the symmetric groups, see [5].

2. General remarks. We begin with a few general remarks concerning the hook representations.

Proposition 1. If $K$ is a field of characteristic not equal to 2 , then $S(r, n)$ is an indecomposable $\Phi_{n}$-module.

Indeed the Specht modules $S^{(\lambda)}$ are all indecomposable over a field of characteristic not equal to 2. One proves that $\operatorname{Hom}_{\Phi_{n}}\left(S^{(\lambda)}, S^{(\lambda)}\right) \cong K$. 
For the rest of this section, $K$ is an arbitrary field. We write $x_{i} \leqq x_{j}$ if $i \leqq j$, and $\left\{y_{1}, \ldots, y_{r}\right\}$ will denote a subset of the set $\left\{x_{1}, \ldots, x_{n}\right\}$.

Proposition 2. A $K$-basis for $S(r, n)$ is given by

Thus

$$
X=\left\{\Delta\left(x_{1}, y_{1}, \ldots, y_{r}\right): x_{1}<y_{1}<\ldots<y_{r} \leqq x_{n}\right\} \text {. }
$$

$$
\operatorname{dim}_{\mathrm{K}} S(r, n)=\left(\begin{array}{c}
n-1 \\
r
\end{array}\right)
$$

This is a special case of the result that a $K$-basis for $S^{(\lambda)}$ is given by $\left\{f^{(\lambda)}(y): y\right.$ is a standard tableau of $(\lambda)\}$ (standard tableaux are defined in [5], to which the reader is referred for the meaning of any undefined terms). The proof that the set $X$ is linearly independent over $K$ is by induction on $n$. $S(r, n)$ is generated over $K$ by the polynomials of the form $\Delta\left(y_{1}, \ldots, y_{r+1}\right)$, $x_{1} \leqq y_{1}<\ldots<y_{r+1} \leqq x_{n}$. The formula

$$
\Delta\left(y_{1}, y_{2}, \ldots, y_{r+1}\right)=\sum_{s=1}^{r+1}(-1)^{s-1} \Delta\left(x_{1}, y_{1}, \ldots, \hat{y}_{s}, \ldots, y_{r+1}\right),
$$

where $\hat{y}_{s}$ is meant to indicate that $y_{s}$ is missing from the sequence $\left(x_{1}, y_{1}, \ldots, y_{s}, \ldots, y_{r+1}\right)$, shows that $X$ generates $S(r, n)$ over $K$. This formula can be proved by expressing the difference products as Vandermonde determinants, and noting that the determinant

$$
\left|\begin{array}{cccc}
1 & 1 & \ldots & 1 \\
1 & 1 & \ldots & 1 \\
x_{1} & y_{1} & \ldots & y_{r+1} \\
\vdots & \vdots & & \vdots \\
x_{1}^{r} & y_{1}^{r} & \ldots & y_{r+1}^{r}
\end{array}\right|
$$

is zero.

The partitions $\left(n-r, 1^{r}\right)$ and $\left(r+1,1^{n-r-1}\right), 0 \leqq r \leqq n-1$, are conjugate in the sense of [5, p. 36]. Write $\Delta=\Delta\left(x_{1}, \ldots, x_{n}\right)$. Then $K \Delta=S(n-1, n)$.

PROPOSITION 3. $S(r, n)$ and $K \Delta \otimes_{K} S(n-r-1, n)$ have the same composition factors, counted according to multiplicity.

This again is a special case of a general result. Let $(\lambda)$ and $\left(\lambda^{\prime}\right)$ be conjugate partitions with corresponding ordinary irreducible characters $\zeta^{(\lambda)}$ and $\zeta^{\left(\lambda^{\prime}\right)}$ respectively. Then $\zeta^{(\lambda)}=\zeta^{\left(1^{n}\right)} \zeta^{\left(\lambda^{\prime}\right)}$, where $\zeta^{\left(1^{n}\right)}$ is the alternating character. This is the basis of the result that $S^{(\lambda)}$ and $K \Delta \otimes_{K} S^{\left(\lambda^{\prime}\right)}$ have the same composition factors.

The final proposition of this section will be of use in Section 5.

Proposition 4. Let $M$ be an indecomposable $\Phi_{n}$-module with a composition series of the form $0 \subset M_{1} \subset M$. Then $M$ has exactly one proper submodule.

Proof. Let $0 \subset M_{1} \subset M$ be a composition series for $M$, and suppose that $N$ is a proper $\Phi_{n}$-submodule of $M$. The chain $0 \subset N \subset M$ can be refined to a composition series, and by the 
Jordan-Hölder Theorem, it must be a composition series. If $M_{1} \cap N=0$, then $M_{1}+N$ (direct sum) would be a submodule of $M$ properly containing $M_{1}$. It would follow that $M=M_{1}+N$ (direct sum), contradicting the assumption that $M$ is indecomposable. Thus $M_{1} \cap N \neq 0$. Since $M_{1}$ and $N$ are irreducible, $M_{1}=M_{1} \cap N=N$, proving that $M_{1}$ is the unique proper submodule of $M$.

3. The case $p$ divides $n$. The results of this section form part of the author's Ph.D Thesis [4]. Throughout this section we assume that the characteristic $p$ of the field $K$ divides $n$. We set

$$
s_{i}=\sum_{k=1}^{n} x_{k}^{i}, \quad i=1,2, \ldots
$$

Suppose that $r<n-1$. Proposition 1 gives a $K$-basis for $S(r, n)$. Using this basis, we define a linear transformation $\theta^{r}: S(r, n) \rightarrow S(r+1, n)$ as follows:

$$
\begin{aligned}
\theta^{r}\left(\Delta\left(x_{1}, y_{1}, \ldots, y_{r}\right)\right) & =\sum_{k=1}^{n} \Delta\left(x_{1}, y_{1}, \ldots, y_{r}, x_{k}\right) \\
& =\left|\begin{array}{ccccc}
1 & 1 & \ldots & 1 & n \\
x_{1} & y_{1} & \ldots & y_{r} & s_{1} \\
\vdots & \vdots & & \vdots & \vdots \\
x_{1}^{r+1} & y_{1}^{r+1} & \ldots & y_{r}^{r+1} & s_{r+1}
\end{array}\right|,
\end{aligned}
$$

extending to the whole of $S(r, n)$ by $K$-linearity. We claim that $\theta^{r}$ is a $\Phi_{n}$-homomorphism. To see this, let $\left\{y_{1}, \ldots, y_{r+1}\right\} \subseteq\left\{x_{1}, \ldots, x_{n}\right\}$ with $y_{1}<y_{2}<\ldots<y_{r+1}$. From (1), we have

$$
\theta\left(\Delta\left(y_{1}, \ldots, y_{r+1}\right)\right)=-\left|\begin{array}{ccccc}
1 & 1 & \ldots & 1 & 0 \\
1 & 1 & \ldots & 1 & n \\
x_{1} & y_{1} & \ldots & y_{r+1} & s_{1} \\
\vdots & \vdots & & \vdots & \vdots \\
x_{1}^{r+1} & y_{1}^{r+1} & \ldots & y_{r+1}^{r+1} & s_{r+1}
\end{array}\right|+\left|\begin{array}{cccc}
1 & \ldots & 1 & n \\
y_{1} & \ldots & y_{r+1} & s_{1} \\
\vdots & & \vdots & \vdots \\
y_{1}^{r+1} & \ldots & y_{r+1}^{r+1} & s_{r+1}
\end{array}\right| .
$$

Since $p$ divides $n$,

$$
\theta^{r}\left(\Delta\left(y_{1}, \ldots, y_{r+1}\right)\right)=\left|\begin{array}{cccc}
1 & \ldots & 1 & n \\
y_{1} & \ldots & y_{r+1} & s_{1} \\
\vdots & & \vdots & \vdots \\
y_{1}^{r+1} & \ldots & y_{r+1}^{r+1} & s_{r+1}
\end{array}\right| .
$$

From this it follows that $\theta^{r}$ is a $\Phi_{n}$-homomorphism. 
Thus we have a chain of $\Phi_{n}$-modules and $\Phi_{n}$-homomorphisms:

$$
0 \rightarrow S(0, n) \stackrel{\theta^{0}}{\rightarrow} S(1, n) \stackrel{\theta^{1}}{\rightarrow} \ldots \rightarrow S(n-2, n) \stackrel{\theta^{n-2}}{\rightarrow} S(n-1, n) \rightarrow 0 .
$$

We proceed to investigate the properties of this sequence.

Certainly $S(r, n-1)$ is a $\Phi_{n-1}$-submodule of $S(r, n)$. Let $\phi^{r}$ denote the restriction of $\theta^{r}$ to this submodule. Then $\phi^{r}$ is a $\Phi_{n-1}$-homomorphism.

Lemma $1 . \operatorname{Im} \theta^{r}=\operatorname{Im} \phi^{r}$.

Proof. All we need to show is that $\operatorname{Im} \theta^{r} \subseteq \operatorname{Im} \phi^{r}$. We do this by checking that $\operatorname{\theta r}\left(\Delta\left(x_{1}, y_{1}, \ldots, y_{r}\right)\right) \in \operatorname{Im} \phi^{r}$ for all $y_{1}, \ldots, y_{r}$ with $x_{1}<y_{1}<\ldots<y_{r} \leqq x_{n}$. Clearly it is sufficient to take $y_{r}=x_{n}$.

$$
\begin{aligned}
\sum_{k=1}^{n-1} \theta^{r}\left(\Delta\left(x_{1}, y_{1}, \ldots, y_{r-1}, x_{k}\right)\right) & =\sum_{k, l=1}^{n} \Delta\left(x_{1}, y_{1}, \ldots, y_{r-1}, x_{k}, x_{l}\right)-\sum_{l=1}^{n} \Delta\left(x_{1}, y_{1}, \ldots, y_{r-1}, x_{n}, x_{l}\right) \\
& =-\theta^{r}\left(\Delta\left(x_{1}, y_{1}, \ldots, y_{r-1}, x_{n}\right)\right) .
\end{aligned}
$$

Lemma 1 follows from this.

For the rest of this section, we assume that $0<r<n-1$.

Lemma 2. Suppose that $S(r, n-1)$ and $S(r-1, n-1)$ are irreducible $\Phi_{n-1}$-modules. Then

$$
\operatorname{Im} \theta^{r-1}=\operatorname{ker} \theta^{r}
$$

Proof. $\theta^{r} \theta^{r-1}\left(\Delta\left(y_{1}, \ldots, y_{r}\right)\right)=\sum_{k, l=1}^{n} \Delta\left(y_{1}, \ldots, y_{r}, x_{k}, x_{l}\right)=0 . \quad$ Hence $\operatorname{Im} \theta^{r-1} \subseteq \operatorname{ker} \theta^{r}$. We prove the reverse inclusion by counting dimensions.

By hypothesis, $S(r, n-1)$ is an irreducible $\Phi_{n-1}$-module, and certainly $\phi^{r} \neq 0$. Hence $\operatorname{ker} \phi^{r}=0$. Consequently, by Lemma 1 and Proposition 1 ,

$$
\operatorname{dim}_{K} \operatorname{Im} \theta^{r}=\operatorname{dim}_{K} \operatorname{Im} \phi^{r}=\operatorname{dim}_{K} S(r, n-1)=\left(\begin{array}{c}
n-2 \\
r
\end{array}\right) .
$$

Therefore

$$
\operatorname{dim}_{K} \operatorname{ker} \theta^{r}=\operatorname{dim}_{K} S(r, n)-\operatorname{dim}_{K} \operatorname{Im} \theta^{r}=\left(\begin{array}{c}
n-1 \\
r
\end{array}\right)-\left(\begin{array}{c}
n-2 \\
r
\end{array}\right)=\left(\begin{array}{c}
n-2 \\
r-1
\end{array}\right) .
$$

By hypothesis, $S(r-1, n-1)$ is irreducible; whence $\operatorname{ker} \phi^{r-1}=0$. Hence by Lemma 1 and Proposition 1,

$$
\operatorname{dim}_{K} \operatorname{Im} \theta^{r-1}=\operatorname{dim}_{K} \operatorname{Im} \phi^{r-1}=\operatorname{dim}_{K} S(r-1, n-1)=\left(\begin{array}{c}
n-2 \\
r-1
\end{array}\right) .
$$

Thus $\operatorname{dim}_{K} \operatorname{Im} \theta^{r-1}=\operatorname{dim}_{K} \operatorname{ker} \theta^{r}$, and this completes the proof of Lemma 2 . 
THEOREM 1. Assuming that $p$ divides $n$, let $0<r<n-1$, and suppose that $S(r, n-1)$ and $S(r-1, n-1)$ are irreducible $\Phi_{n-1}$-modules. Then a composition series for $S(r, n)$ is given by

$$
0 \subset \operatorname{ker} \theta^{r} \subset S(r, n),
$$

where $\theta^{r}$ is the $\Phi_{n}$-homomorphism (2). Further, $\operatorname{ker} \theta^{r} \cong S(r-1, n-1)$ over $\Phi_{n-1}$.

Proof. By Lemmas 1 and 2, there are $\Phi_{n-1}$-isomorphisms

$$
\operatorname{ker} \theta^{r}=\operatorname{Im} \theta^{r-1}=\operatorname{Im} \phi^{r-1} \cong S(r-1, n-1)
$$

because $\operatorname{ker} \phi^{r-1}=0$. It follows that $\operatorname{ker} \theta^{r}$ is irreducible as a $\Phi_{n-1}$-module, and hence as a $\Phi_{n}$-module. Also there are $\Phi_{n-1}$-isomorphisms

$$
\frac{S(r, n)}{\operatorname{ker} \theta^{r}} \cong \operatorname{Im} \theta^{r}=\operatorname{Im} \phi^{r} \cong S(r, n-1)
$$

The irreducibility of $S(r, n-1)$ over $\Phi_{n-1}$ implies that of $S(r, n) / \operatorname{ker} \theta^{r}$ over $\Phi_{n}$.

COROLlary. Take $n=p$. Let $\theta^{r}$ be the homomorphism (2). Then if $0<r<p-1$, a composition series for $S(r, p)$ is given by

$$
0 \subset \operatorname{ker} \theta^{r} \subset S(r, p) .
$$

Proof. This follows immediately because we know that $S(r, p-1)$ is irreducible for all $r$.

REMARKS. By Lemma 2, the sequence (3) is exact in the case in which $n=p$; thus if $r<p-2$,

$$
\frac{S(r, p)}{\operatorname{ker} \theta^{r}} \cong \operatorname{Im} \theta^{r}=\operatorname{ker} \theta^{r+1}
$$

Hence if $0 \leqq r \leqq p-2, S(r, p)$ and $S(r+1, p)$ have a common composition factor. Further, Proposition 3 says that $S(r-1, p)$ and $K \Delta \otimes_{K} S(p-r, p)$ have the same composition factors. A composition series for the latter is given by

$$
0 \subset K \Delta \otimes_{K} \operatorname{ker} \theta^{p-r} \subset K \Delta \otimes_{K} S(p-r, p) .
$$

Counting dimensions we see that

$$
K \Delta \otimes_{K} \operatorname{ker} \theta^{p-r} \cong \frac{S(r-1, p)}{\operatorname{ker} \theta^{r-1}} \cong \operatorname{ker} \theta^{r}
$$

We cannot have $\operatorname{ker} \theta^{r} \cong \operatorname{ker} \theta^{s}$ with $r \neq s$, since

$$
\left(\begin{array}{l}
p-2 \\
r-1
\end{array}\right)=\operatorname{dim}_{K} \operatorname{ker} \theta^{r}=\operatorname{dim}_{K} \operatorname{ker} \theta^{s}=\left(\begin{array}{l}
p-2 \\
s-1
\end{array}\right)
$$

can only occur when $r=p-s$, and when this is satisfied, the relationship between $\operatorname{ker} \theta^{r}$ and $\operatorname{ker} \theta^{s}$ is as described.

These results give the modular representation theory of $S_{p}$ over the field of $p$ elements as obtained by T. Nakayama [2, Theorems 2 and 4], and R. M. Thrall and C. Nesbitt [6]. The 
Specht modules $S^{(\lambda)}$, where $(\lambda)$ is a partition of $p$ whose diagram is not a hook, together with the $\operatorname{ker} \theta^{r}, r=0,1, \ldots, p-2$, and $K \Delta$ constitute a full set of irreducible, non-isomorphic left $\Phi_{p}$-modules.

H. K. Farahat [1, Theorem 5.2] proves that $S(1, n)$ is irreducible if $p$ does not divide $n$. Further, taking $p$ to be a factor of $n$, he obtains the same composition series for $S(1, n)$ as is given by applying Theorem 1 with $r=1$. In [4], we prove that $S(2, n)$ is irreducible if $p$ does not divide $n$ and $p \neq 2$. In Section 5 we prove the conjecture made in [4] that $S(r, n)$ is irreducible if $p$ does not divide $n$ and $p \neq 2$. The composition factors of $S(r, n)$ in the case when $p$ divides $n$ are then as in Theorem 1. We shall see in Section 6 that if the ground field has characteristic 2, the hook representations are reducible, except for $r=0, n-1$, and possibly $r=1, n-2$. In this situation, Theorem 1 is not applicable.

3. Restriction to $S_{n-1}$. In preparation for the analysis of the hook representations by induction on $n$, we examine the structure of $S(r, n)$ regarded as a $\Phi_{n-1}$-module. In this section, $K$ is an arbitrary field and $0<r<n-1$.

Using the basis of $S(r, n)$ given by Proposition 2, we define a linear transformation $\psi r: S(r, n) \rightarrow S(r-1, n-1)$ as follows:

$$
\psi^{r}\left(\Delta\left(x_{1}, y_{1}, \ldots, y_{r}\right)\right)= \begin{cases}\Delta\left(x_{1}, y_{1}, \ldots, y_{r-1}\right), & x_{1}<y_{1}<\ldots<y_{r-1}<y_{r}=x_{n} \\ 0, & x_{1}<y_{1}<\ldots<y_{r-1}<y_{r}<x_{n},\end{cases}
$$

extending to the whole of $S(r, n)$ by $K$-linearity. Using (1), it is clear that $\psi^{r}$ is a $\Phi_{n-1}$-homomorphism. Obviously $\psi^{r}$ is an epimorphism. Note also that $S(r, n-1)$ is a $\Phi_{n-1}$-submodule of $S(r, n)$ contained in $\operatorname{ker} \psi^{r}$.

LEMMA 3. The following sequence of $\Phi_{n-1}$-modules is exact:

$$
0 \rightarrow S(r, n-1) \stackrel{\text { incl. }}{\longrightarrow} S(r, n) \stackrel{\psi r}{\longrightarrow} S(r-1, n-1) \rightarrow 0 .
$$

Proof. We have $S(r, n-1) \subseteq \operatorname{ker} \psi^{r}$. It follows by counting dimensions that

$$
S(r, n-1)=\operatorname{ker} \psi^{r} \text {. }
$$

For the moment, let $S_{n-2}$ be the group of permutations of the set $\left\{x_{2}, \ldots, x_{n-1}\right\}$. The map $\omega_{0}^{r}: S(r-1, n-1) \rightarrow S(r, n)$ defined by

$$
\omega_{0}^{r}\left(\Delta\left(x_{1}, y_{1}, \ldots, y_{r-1}\right)\right)=\Delta\left(x_{1}, y_{1}, \ldots, y_{r-1}, x_{n}\right) \quad x_{1}<y_{1}<\ldots<y_{r-1}<x_{n},
$$

is a $\Phi_{n-2}$-homomorphism, where $\Phi_{n-2}$ is the group algebra of $S_{n-2}$ with coefficients in $K$. Clearly $\psi^{r} \omega_{0}^{r}$ is the identity map on $S(r-1, n-1)$. Suppose that $p$ does not divide $n-1$, and define $\omega^{r}: S(r-1, n-1) \rightarrow S(r, n)$ by

$$
\omega^{r}(z)=(n-1)^{-1} \sum_{k=1}^{n-1}\left(x_{1} x_{k}\right) \omega_{0}^{r}\left(\left(x_{1} x_{k}\right) z\right) \quad(z \in S(r-1, n-1)) .
$$

Then $\omega^{r}$ is a $\Phi_{n-1}$-homomorphism, and $\psi^{r} \omega^{r}$ is the identity map on $S(r-1, n-1)$. This means that the exact sequence (4) splits; thus $\operatorname{Im} \omega^{r} \cong S(r-1, n-1)$ and $S(r, n)=S(r, n-1)+\operatorname{Im} \omega^{r}$, (direct sum). This proves 
LEMMA 4. If the characteristic of the field $K$ does not dividen-1, there is a $\Phi_{n-1}$-isomorphism

$$
S(r, n) \cong S(r-1, n-1)+S(r, n-1) \quad \text { (direct sum). }
$$

5. Analysis of hook representations. The one theorem of this section gives the composition factors of the hook representations when the ground field has characteristic $p$ not equal to 2 .

THEOREM 2. Let $K$ be a field of characteristic $p$ not equal to 2. If $p$ does not divide $n$, $S(r, n)$ is irreducible for each $r, 0 \leqq r \leqq n-1$. If $p$ divides $n$, a composition series for $S(r, n)$, $0<r<n-1$, is given by

$$
0 \subset \operatorname{ker} \theta^{r} \subset S(r, n),
$$

where $\theta^{r}: S(r, n) \rightarrow S(r+1, n)$ is the $\Phi_{n}$-homomorphism (2). $S(0, n)$ and $S(n-1, n)$ are of course still irreducible when $p$ divides $n$.

Proof. Throughout the proof we assume that $0<r<n-1$ and $n \geqq 3$. We proceed by induction on $n$.

Induction hypothesis: $p \nmid n$ implies that $S(r, n)$ is irreducible for each $r, 0<r<n-1$; $p \mid n$ implies that each $S(r, n)$ has a composition series of the form $0 \subset M \subset S(r, n)$.

The remarks at the end of Section 3, together with Proposition 3, are sufficient to cover the cases when $n \leqq 6$. Suppose that $n>3$, and that the induction hypothesis holds for smaller values of the inductive argument. The proof is divided into three parts.

(a) $p \mid n$. In this case, $p$ does not divide $n-1$, and so $S(r, n-1)$ and $S(r-1, n-1)$ are irreducible $\Phi_{n-1}$-modules by the induction hypothesis. By Theorem $1, S(r, n)$, has a composition series

$$
0 \subset \operatorname{ker} \theta^{r} \subset S(r, n)
$$

where $\theta^{r}$ is the $\Phi_{n}$-homomorphism (2).

(b) $p \nmid n, p \nmid n-1$. By Lemma 4, there is a $\Phi_{n-1}$-isomorphism

$$
S(r, n) \cong S(r, n-1)+S(r-1, n-1) \quad \text { (direct sum). }
$$

Thus, by the induction hypothesis $S(r, n)$ is completely reducible as a $\Phi_{n-1}$-module. Let $X$ be a $\Phi_{n}$-submodule of $S(r, n)$. Then $X$ is a $\Phi_{n-1}$-submodule of $S(r, n)$, and as such is a direct summand. Hence there exists a $\Phi_{n-1}$-homomorphism $\eta_{0}: S(r, n) \rightarrow X$ such that the restriction of $\eta_{0}$ to $X$ is the identity map on $X$, namely the projection onto $X$. We are supposing that $p$ does not divide $n$, so that $n^{-1}$ exists in $K$. We can therefore define $\eta: S(r, n) \rightarrow X$ by

$$
\eta(z)=n^{-1} \sum_{i=1}^{n}\left(x_{i} x_{n}\right) \eta_{0}\left(\left(x_{i} x_{n}\right) z\right) \quad(z \in S(r, n)) .
$$

Then $\eta$ is a $\Phi_{n}$-homomorphism, and the restriction of $\eta$ to $X$ is the identity map on $X$. It follows that $S(r, n)=X+\operatorname{Im} \eta$, (direct sum), where both $X$ and $\operatorname{Im} \eta$ are $\Phi_{n}$-submodules of $S(r, n)$. But $S(r, n)$ is indecomposable according to Proposition 1; whence $X=0$ or $X=S(r, n)$. Thus $S(r, n)$ is irreducible.

(c) $p \nmid n, p \mid n-1$. In Lemma 3 we found the following exact sequence of $\Phi_{n-1}$-modules:

$$
0 \rightarrow S(r, n-1) \rightarrow S(r, n) \stackrel{\psi^{r}}{\rightarrow} S(r-1, n-1) \rightarrow 0 \text {. }
$$


Since $p$ divides $n-1$, there exist $\Phi_{n-1}$-homomorphisms $\theta^{t}: S(t, n-1) \rightarrow S(t+1, n-1)$, $0 \leqq t \leqq n-2 ; \operatorname{Im} \theta^{t-1}=\operatorname{ker} \theta^{t}$ for $0<t \leqq n-2$, as in Section 3. By the induction hypothesis, each of $\operatorname{ker} \theta^{r}, S(r, n-1) / \operatorname{ker} \theta^{r}, \operatorname{ker} \theta^{r-1}$ and $S(r-1, n-1) / \operatorname{ker} \theta^{r-1}$ is an irreducible $\Phi_{n-1}$-module.

From the above exact sequence of $\Phi_{n-1}$-modules, we can construct a chain of $\Phi_{n-1}$-submodules of $S(r, n)$ of the form

$$
0 \subset X_{1} \subseteq X_{2} \subseteq X_{3} \subset S(r, n),
$$

where $X_{2}=S(r, n-1)$ and $X_{1}=\operatorname{ker} \theta^{r}:$ in the case in which $r=n-2$, we have $X_{2} \cong K \Delta\left(x_{1}, \ldots, x_{n-1}\right)$, and $X_{1}=X_{2}$; otherwise $X_{1}$ is a proper submodule of $X_{2}$. Also the map $\psi^{r}$ induces a $\Phi_{n-1}$-isomorphism

$$
\bar{\psi}^{r}: \frac{S(r, n)}{X_{2}} \rightarrow S(r-1, n-1)
$$

and $X_{3}$ is the $\Phi_{n-1}$-submodule of $S(r, n)$ containing $X_{2}$ and corresponding to $\operatorname{ker} \theta^{-1}$; thus

$$
\Psi^{r}: \frac{X_{3}}{X_{2}} \rightarrow \operatorname{ker} \theta^{r-1}=\operatorname{Im} \theta^{r-2}
$$

is a $\Phi_{n-1}$-isomorphism: in the case in which $r=1$, we have $S(r, n) / X_{2} \cong K$, and $X_{3}=X_{2}$; in all other cases $X_{2} \subset X_{3}$.

At this point we construct a specific submodule $X_{3}$ which has the properties just described. We take $r>1$. Propositions 1 and 4 show that there is exactly one proper $\Phi_{n-1}$-submodule of $S(r, n)$ containing $X_{2}$ properly.

Let $X_{3}$ be the smallest $\Phi_{n-1}$-submodule of $S(r, n)$ containing $X_{2}$ and all polynomials of the form

$$
\sum_{k=1}^{n-1} \Delta\left(y_{1}, \ldots, y_{r-1}, x_{k}, x_{n}\right), \quad x_{1} \leqq y_{1}<\ldots<y_{r-1}<x_{n} .
$$

By (1), and using the assumption that $p$ divides $n-1$, we have

$$
\begin{aligned}
\sum_{k=1}^{n-1} \Delta\left(y_{1}, \ldots, y_{r-1}, x_{k}, x_{n}\right)= & \sum_{k=1}^{n-1} \sum_{l=1}^{r-1}(-1)^{l-1} \Delta\left(x_{1}, y_{1}, \ldots, \mathfrak{y}_{l}, \ldots, y_{r-1}, x_{k}, x_{n}\right) \\
& +(-1)^{r} \sum_{k=1}^{n-1} \Delta\left(x_{1}, y_{1}, \ldots, y_{r-1}, x_{k}\right) .
\end{aligned}
$$

Further (if $r>2$ ),

$$
\sum_{l=1}^{n-1} \sum_{k=1}^{n-1} \Delta\left(x_{1}, y_{1}, \ldots, y_{r-3}, x_{l}, x_{k}, x_{n}\right)=0
$$

It follows that $X_{3}$ is generated over $K$ by the set

$$
\begin{aligned}
& \left\{\Delta\left(x_{1}, y_{1}, \ldots, y_{r}\right): x_{1}<y_{1}<\ldots<y_{r}<x_{n}\right\} \cup\left\{\sum_{k=1}^{n-1} \Delta\left(x_{1}, y_{1}, \ldots, y_{r-2}, x_{k}, x_{n}\right):\right. \\
& \left.x_{1}<y_{1}<\ldots<y_{r-2}<x_{n-1}\right\} \text {. }
\end{aligned}
$$


(In the case in which $r=2$, the second set in this union consists of the element $\sum_{k=1}^{n-1} \Delta\left(x_{1}, x_{k}, x_{n}\right)$ alone.) It is easy to check that this set is linearly independent over $K$ because

$$
\left\{\Delta\left(x_{1}, y_{1}, \ldots, y_{r}\right): x_{1}<y_{1}<\ldots<y_{r} \leqq x_{n}\right\}
$$

is linearly independent over $K$. We therefore have a $K$-basis for $X_{3}$ which yields a $K$-basis for $X_{3} / X_{2}$. We have now constructed a $\Phi_{n-1}$-composition series (5) for $S(r, n)$.

Let $M$ be a non-zero $\Phi_{n}$-submodule of $S(r, n)$, and consider the $\Phi_{n-1}$-submodule $Y=M \cap X_{2}$. There are three possibilities: $Y=0,0 \subset Y \subset X_{2}$, or $Y=X_{2}$. In the third case, $X_{2} \subseteq M$; whence $\Delta\left(x_{1}, \ldots, x_{r+1}\right) \in M$. Since $M$ is a $\Phi_{n}$-submodule of $S(r, n)$, this implies that $M=S(r, n)$. We shall prove that the other conditions are impossible by deducing a contradiction from each. It will follow from this that $S(r, n)$ is an irreducible $\Phi_{n}$-module,

First suppose that $0 \subset Y \subset X_{2}$ (this does not arise if $r=n-2$ ). By Propositions 1 and 4, $Y=X_{1}$. It follows that $X_{1} \subseteq M$. Now $X_{1}=\operatorname{ker} \theta^{r}=\operatorname{Im} \theta^{r-1}$, and hence $M$ contains

$$
\theta^{r-1}\left(\Delta\left(x_{1}, x_{2}, \ldots, x_{r}\right)\right)=\sum_{k=1}^{n-1} \Delta\left(x_{1}, x_{2}, \ldots, x_{r}, x_{k}\right) .
$$

Applying the permutation $\left(x_{n} x_{n-1}\right)$ to this element gives

$$
\sum_{\substack{k=1 \\ k \neq n=1}}^{n} \Delta\left(x_{1}, x_{2}, \ldots, x_{r}, x_{n-1}\right)
$$

The difference of these two, namely,

$$
\Delta\left(x_{1}, \ldots, x_{r}, x_{n}\right)-\Delta\left(x_{1}, \ldots, x_{r}, x_{k}\right)
$$

belongs to $M$. Applying $\left(x_{r}, x_{n}\right)$ to (6) and adding the result to (6), we find that

$$
-\Delta\left(x_{1}, \ldots, x_{r-1}, x_{n}, x_{n-1}\right)-\Delta\left(x_{1}, \ldots, x_{r-1}, x_{r}, x_{n-1}\right)
$$

belongs to $M$. Apply $\left(x_{r} x_{n-1}\right)$ to this element and add the result to (6); this yields $2 \Delta\left(x_{1}, \ldots, x_{r-1}, x_{r}, x_{n}\right) \in M$. Since $p \neq 2, \Delta\left(x_{1}, \ldots, x_{r}, x_{n}\right) \in M$, whence $M=S(r, n)$. This contradicts $Y=M \cap X_{2} \subset X_{2}$.

For the final part of the proof, suppose that $Y=0$. Then $M+X_{2}$ (direct sum) is a $\Phi_{n-1}$-submodule of $S(r, n)$ containing $X_{2}$ properly. Suppose first that $M+X_{2}$ is a proper submodule of $S(r, n)$ (if $r=1$, this situation does not arise). Then, by Propositions 1 and 4,

$$
\frac{M+X_{2}}{X_{2}}=\frac{X_{3}}{X_{2}}
$$

i.e. $M+X_{2}=X_{3}$. Let $q: X_{3} \rightarrow X_{2}$ be the projection onto $X_{2}=S(r, n-1)$, and write $\sum_{k=1}^{n-1} \Delta\left(x_{1}, \ldots, x_{r-1}, x_{k}, x_{n}\right)=\xi \in X_{3}$. Since $q(\xi) \in X_{2}$, we have

$$
q(\xi)=\sum_{1<i_{1}<\ldots<i_{r}<n} \lambda_{i_{1} \ldots i_{r}} \Delta\left(x_{1}, x_{i_{1}}, \ldots, x_{i_{r}}\right),
$$


where each $\hat{\lambda}_{i_{1} \ldots i_{r}} \in K$. In each sequence $\left(i_{1}, \ldots, i_{r}\right)$ with $1<i_{1}<\ldots<i_{r}<n$, both $i_{r}>r-1$ and $i_{r-1}>r-1$. Since $\left(x_{i_{r}} x_{i_{r-1}}\right) \xi=\xi$, and because $q$ is a $\Phi_{n-1}$-homomorphism, we have $\left(x_{i_{r}} x_{i_{r-1}}\right) q(\xi)=q(\xi)$. Equating coefficients of $\Delta\left(x_{1}, x_{i_{1}}, \ldots, x_{i_{r}}\right)$, we have $\lambda_{i_{1} \ldots i_{r}}=0$. Thus $q(\xi)=0$, and this means that $\xi \in M$, i.e.

$$
\sum_{k=1}^{n-1} \Delta\left(x_{1}, \ldots, x_{r-1}, x_{k}, x_{n}\right) \in M
$$

Apply the permutation $\left(x_{1}, x_{l}\right)$ to this element, $l \neq 1,2, \ldots, r-1, n$. We have by (1)

$$
\begin{aligned}
\sum_{k=1}^{n-1} \Delta\left(x_{l}, x_{2}, \ldots, x_{r-1}, x_{k}, x_{n}\right)= & \sum_{k=1}^{n-1} \Delta\left(x_{1}, x_{2}, \ldots, x_{r-1}, x_{k}, x_{n}\right) \\
& +\sum_{k=1}^{n-1} \sum_{s=2}^{r-1}(-1)^{s-1} \Delta\left(x_{1}, x_{l}, x_{2}, \ldots, x_{s}, \ldots, x_{r-1}, x_{k}, x_{n}\right) \\
& +(n-1)(-1)^{r-1} \Delta\left(x_{1}, x_{l}, x_{2}, \ldots, x_{r-1}, x_{n}\right) \\
& +(-1)^{r} \sum_{k=1}^{n-1} \Delta\left(x_{1}, x_{l}, x_{2}, \ldots, x_{r-1}, x_{k}\right) .
\end{aligned}
$$

Since $p$ divides $n-1, \sum_{k=1}^{n-1} \Delta\left(x_{1}, x_{2}, \ldots, x_{r-1}, x_{k}\right) \in M$. This element also belongs to $S(r, n-1)$, contradicting $M \cap S(r, n-1)=0$.

This rules out the possibility that $M+X_{2}$ is a proper submodule of $S(r, n)$ i.e. $M+X_{2}=S(r, n)$. Let $q_{1}: S(r, n) \rightarrow M$ be the projection onto $M$. Then $q_{1}$ is a $\Phi_{n-1}$-homomorphism. Define $q: S(r, n) \rightarrow M$ by

$$
q(z)=n^{-1} \sum_{k=1}^{n}\left(x_{n} x_{k}\right) q_{1}\left(\left(x_{n} x_{k}\right) z\right) \quad(z \in S(r, n)) .
$$

Then $q$ is a $\Phi_{n}$-homomorphism, and $S(r, n)=M+\operatorname{Im} q$ (direct sum). This contradicts Proposition 1, and completes the proof of Theorem 2.

6. Hook representations and natural representations at characteristic 2 . We begin this section by defining the natural representation modules. These are studied with a view to constructing irreducible and indecomposable representation modules of the symmetric groups.

Let $K$ be an arbitrary field. The $\Phi_{n}$-module $M^{r}(n)$ defined by

$$
\begin{aligned}
& M^{r}(n)=\Phi_{n} x_{1} \ldots x_{r} \quad(0<r \leqq n), \\
& M^{\circ}(n)=K,
\end{aligned}
$$

is called the $r$ th natural representation module of $S_{n}$ (over $K$ ). A $K$-basis for $M^{r}(n)$ is given by

$$
\left\{y_{1} \ldots y_{r}: x_{1} \leqq y_{1}<\ldots<y_{r} \leqq x_{n}\right\} .
$$

Thus $\operatorname{dim}_{R} M^{r}(n)=\left(\begin{array}{l}n \\ r\end{array}\right)$. For example, $M^{n}(n) \cong K=M^{0}(n)$. 
Define the map $\omega_{r}: M^{r}(n) \rightarrow M^{n-r}(n)$ by

$$
\omega_{r}\left(\sigma x_{1} \ldots x_{r}\right)=\sigma x_{r+1} \ldots x_{n} \quad\left(\sigma \in \Phi_{n}\right)
$$

It is easy to check that $\omega_{r}$ is a well defined $\Phi_{n}$-isomorphism, so that $M^{r}(n) \cong M^{n-r}(n)$. Thus, in studying the natural representation modules, it is sufficient to treat only the cases when $2 r \leqq n$.

If $2 r \leqq n,(n-r, r)$ is a partition of $n$. We denote by $S^{r}(n)$ the corresponding Specht module. Thus $S^{r}(n)$ consists of all $K$-linear combinations of polynomials of the form

$$
\left(x_{a_{1}}-x_{b_{1}}\right)\left(x_{a_{2}}-x_{b_{2}}\right) \ldots\left(x_{a_{r}}-x_{b_{r}}\right) \text {, }
$$

where $a_{1}, \ldots, a_{r}, b_{1}, \ldots, b_{r}$ are all distinct. It is clear that $S^{r}(n) \subseteq M^{r}(n)$. A $K$-basis for $S^{\prime}(n)$ can be found in terms of the standard tableaux of the partition $(n-r, r)$.

Let $d: K\left[x_{1}, \ldots, x_{n}\right] \rightarrow K\left[x_{1}, \ldots, x_{n}\right]$ be the map defined in terms of the partial differential operators by

$$
d=\sum_{i=1}^{n} \frac{\partial}{\partial x_{i}}
$$

Restricting to $M^{r+1}(n), r<n$, we obtain a $\Phi_{n}$-homomorphism

$$
d_{r}: M^{r+1}(n) \rightarrow M^{r}(n) \quad(r=0,1, \ldots, n-1) .
$$

We clearly have

$$
d_{r}\left(y_{1} \ldots y_{r+1}\right)=\sum_{j=1}^{r+1} y_{1} \ldots \hat{y}_{j} \ldots y_{r+1},
$$

where $\hat{y}_{j}$ is meant to indicate that $y_{j}$ is missing from the product $y_{1} \ldots y_{r+1}$.

For the rest of this section we assume that $K$ has characteristic 2 . We intend to study the following sequence of $\Phi_{n}$-modules and $\Phi_{n}$-homomorphisms:

$$
0 \rightarrow M^{n}(n) \stackrel{d_{n-1}}{\longrightarrow} M^{n-1}(n) \rightarrow \ldots \rightarrow M^{2}(n) \stackrel{d_{1}}{\rightarrow} M^{1}(n) \stackrel{d_{0}}{\rightarrow} M^{0}(n) \rightarrow 0 .
$$

Clearly $\operatorname{ker} d_{n-1}=0$ and $\operatorname{Im} d_{0}=M^{0}(n)$. We prove that (7) is exact. Obviously, because $K$ has characteristic 2 ,

Thus $\operatorname{Im} d_{r+1} \subseteq \operatorname{ker} d_{r}$.

$$
d_{r} d_{r+1}=0 \quad(r=0,1, \ldots, n-2) .
$$

A $K$-basis for $S(r, n)$ is given by Proposition 2. Using this basis, we define a linear transformation $\eta_{r}: S(r, n) \rightarrow M^{\prime}(n)$ by

$$
\eta_{r}\left(\Delta\left(x_{1}, y_{1}, \ldots, y_{r}\right)\right)=d_{r}\left(x_{1} y_{1} \ldots y_{r}\right)
$$

extending to the whole of $S(r, n)$ by $K$-linearity. By (1)

$$
\begin{aligned}
\eta_{r}\left(\Delta\left(y_{1}, \ldots, y_{r+1}\right)\right) & =\sum_{s=1}^{r+1} d\left(x_{1} y_{1} \ldots \hat{y}_{s} \ldots y_{r+1}\right) \\
& =x_{1} d\left(\sum_{s=1}^{r+1} y_{1} \ldots \hat{y}_{s} \ldots y_{r+1}\right)+\sum_{s=1}^{r+1} y_{1} \ldots \hat{y}_{s} \ldots y_{r+1} \\
& =x_{1} d^{2}\left(y_{1} \ldots y_{r+1}\right)+d\left(y_{1} \ldots y_{r+1}\right) \\
& =d\left(y_{1} \ldots y_{r+1}\right) .
\end{aligned}
$$


It follows that $\eta_{r}$ is a $\Phi_{n}$-homomorphism.

Suppose that

$$
\eta_{r}\left(\sum_{1<i_{1}<\ldots<i_{r} \leqq n} \lambda_{i_{1}, \ldots, i_{r}} \Delta\left(x_{1}, x_{i_{1}}, \ldots, x_{i_{r}}\right)=0,\right.
$$

where each $\lambda_{i_{1}, \ldots, i_{r}} \in K$. Then

i.e.

$$
\sum_{1<i_{1}<\ldots<i_{r} \leqq n} \lambda_{i_{1}, \ldots, i_{r}} d\left(x_{1} x_{i_{1}} \ldots x_{i_{r}}\right)=0,
$$

$$
\sum_{1<i_{1}<\ldots<i_{r} \leqq n} \lambda_{i_{1}, \ldots, i_{r}} x_{i_{1}} \ldots x_{i_{r}}+\sum_{1<i_{1}<\ldots<i_{r} \leqq n} \lambda_{i_{1}, \ldots, i_{r}} x_{1} d\left(x_{i_{1}} \ldots x_{i_{r}}\right)=0 .
$$

Equating coefficients of $x_{i_{1}} \ldots x_{i_{r}}, 1<i_{1}<\ldots<i_{r} \leqq n$, we find that $\lambda_{i_{1}, \ldots, i_{r}}=0$. This proves that

$$
\operatorname{ker} \eta_{r}=0 \text {, }
$$

i.e. $\eta_{r}$ is a monomorphism.

Now define $\alpha_{r}: M^{r+1}(n) \rightarrow S(r, n), r=0,1, \ldots, n-1$, by

$$
\alpha_{r}\left(y_{1} \ldots y_{r+1}\right)=\Delta\left(y_{1}, \ldots, y_{r+1}\right) \quad\left(1 \leqq y_{1}<\ldots<y_{r+1} \leqq n\right),
$$

extending to the whole of $M^{r+1}(n)$ by $K$-linearity. It is clear that $\alpha_{r}$ is a $\Phi_{n}$-homomorphism, and $\operatorname{Im} \alpha_{r}=S(r, n)$, i.e. $\alpha_{r}$ is an epimorphism.

Obviously

$$
d_{r}=\eta_{r} \alpha_{r}
$$

We have thus factorised $d_{r}$ into the composition of an epimorphism and a monomorphism. It follows that $\operatorname{Im} \eta_{r}=\operatorname{Im} d_{r}$ and $\operatorname{ker} \alpha_{r}=\operatorname{ker} d_{r}$. We have already noted that $\operatorname{Im} d_{r+1} \subseteq \operatorname{ker} d_{r}$. We prove the reverse inclusion by counting dimensions. Thus

and

$$
\operatorname{dim}_{K} \operatorname{Im} d_{r+1}=\operatorname{dim}_{K} \operatorname{Im} \eta_{r+1}=\operatorname{dim}_{K} S(r+1, n)=\left(\begin{array}{c}
n-1 \\
r+1
\end{array}\right),
$$

$$
\operatorname{dim}_{K} \operatorname{ker} d_{r}=\operatorname{dim}_{K} M^{r+1}(n)-\operatorname{dim}_{K} \operatorname{Im} d_{r}=\left(\begin{array}{c}
n \\
r+1
\end{array}\right)-\left(\begin{array}{c}
n-1 \\
r
\end{array}\right)=\left(\begin{array}{c}
n-1 \\
r+1
\end{array}\right)
$$

Thus $\operatorname{dim}_{K} \operatorname{Im} d_{r+1}=\operatorname{dim}_{K} \operatorname{ker} d_{r}$, and it follows that $\operatorname{Im} d_{r+1}=\operatorname{ker} d_{r}$. This completes the proof of

THEOREM 3. The sequence (7) of $\Phi_{n}$-modules is exact.

Suppose that $0<2 r \leqq n$. It is clear that $S^{r}(n) \subseteq \operatorname{ker} d_{r-1}=\operatorname{Im} d_{r}$, so that $S^{r}(n) \subseteq \operatorname{Im} \eta_{r}$. We determine $\eta_{r}^{-1}\left(S^{r}(n)\right)$.

Choose integers $a_{1}, \ldots, a_{r}, b_{1}, \ldots, b_{r}$ all different and satisfying $1<a_{i}, b_{j} \leqq n$. Define the element $\Delta^{a_{1}, b_{1}, a_{2}, b_{2}, \ldots, a_{r}, b_{r}} \in S(r, n)$ by

$$
\Delta^{a_{1}, b_{1}, \ldots, a_{r}, b_{r}}=\sum_{c} \Delta\left(x_{1}, x_{c_{1}}, \ldots, x_{c_{r}}\right)
$$


summing over all sequences $c=\left(c_{1}, \ldots, c_{r}\right)$ in which, for each $i$, either $c_{i}=a_{i}$ or $c_{i}=b_{i}$. By the definition of $\eta_{r}$,

$$
\begin{aligned}
\eta_{r}\left(\Delta^{a_{1}, b_{1}, \ldots, a_{r}, b_{r}}\right) & =\sum_{c} \eta_{r}\left(\Delta\left(x_{1}, x_{c_{1}}, \ldots, x_{c_{r}}\right)\right) \\
& =\sum_{c} d\left(x_{1} x_{c_{1}} \ldots x_{c_{r}}\right) \\
& =x_{1} \sum_{c} d\left(x_{c_{1}} \ldots x_{c_{r}}\right)+\sum_{c} x_{c_{1}} \ldots x_{c_{r}} .
\end{aligned}
$$

But

$$
\sum_{c} x_{c_{1}} \ldots x_{c_{r}}=\left(x_{a_{1}}+x_{b_{1}}\right)\left(x_{a_{2}}+x_{b_{2}}\right) \ldots\left(x_{a_{r}}+x_{b_{r}}\right)
$$

Consequently

$$
\eta_{r}\left(\Delta^{a_{1}, b_{1}, \ldots, a_{r}, b_{r}}\right)=\left(x_{a_{1}}+x_{b_{1}}\right)\left(x_{a_{2}}+x_{b_{2}}\right) \ldots\left(x_{a_{r}}+x_{b_{r}}\right) .
$$

Note also that of $k$ is different from $1, a_{1}, \ldots, a_{r}, b_{1}, \ldots, b_{r}$, then

$$
\eta_{r}\left(\sum_{c} \Delta\left(x_{k}, x_{c_{1}}, \ldots, x_{c_{r}}\right)\right)=\left(x_{a_{1}}+x_{b_{1}}\right) \ldots\left(x_{a_{r}}+x_{b_{r}}\right) \text {. }
$$

Thus, since $\eta_{r}$ is a monomorphism,

$$
\Delta^{a_{1}, b_{1}, \ldots, a_{r}, b_{r}}=\sum_{c} \Delta\left(x_{k}, x_{c_{1}}, \ldots, x_{c_{r}}\right) .
$$

We have now proved

THEOREM 4. Let $K$ be a field of characteristic 2 , and choose $r$ such that $0<2 r \leqq n$. Then $S(r, n)$ contains a submodule isomorphic to $S^{\prime}(n)$ over $\Phi_{n}$, namely the $\Phi_{n}$-submodule generated by the elements $\Delta^{a_{1}, b_{1}, \ldots, a_{r}, b_{r}}$.

Take $r=1$. In this case $\Delta^{a_{1}, b_{1}}=\left(x_{a_{1}}+x_{b_{1}}\right)$, and the submodule is $S(1, n)$. Note that $S^{1}(n)=S(1, n)$. If $r>1$, the submodule is a proper submodule. The results of [3] yield the composition factors of $S(2, n)$ when the ground field has characteristic 2 . The problem of analysing the hook representations is equivalent to the problem of analysing the natural representation modules.

\section{REFERENCES}

1. H. K. Farahat, On the natural representation of the symmetric groups, Proc. Glasgow Math. Assoc. 5 (1962), 121-136.

2. T. Nakayama, On some modular properties of irreducible representations of a symmetric group, II, Japanese J. Math. 17 (1941), 165-184.

3. M. H. Peel, On the second natural representation of the symmetric groups, Glasgow Math. J. 10 (1969), 25-37.

4. M. H. Peel, Modular representations of the symmetric groups, Ph.D. Thesis, Sheffield, 1969. 
5. G. de B. Robinson, Representation theory of the symmetric group, Edinburgh University Press, 1961.

6. R. M. Thrall and C. Nesbitt, On the modular representations of the symmetric groups, Math. Ann. 43 (1942), 656-670.

THE UNIVERSITY

SHEFFIELD 